\title{
Estimating DOA and PPS parameters of signal received by ULA in heavy noise environment
}

\author{
Igor Djurović ${ }^{1}$, Marko Simeunović $^{2 *}$, Vladimir V. Lukin ${ }^{3}$
}

\begin{abstract}
Estimation of the direction-of-arrival (DOA) and parameters of polynomial phase signal (PPS) impinging on the uniform linear array (ULA) of sensors in heavy-tailed noise environments is considered in this paper. To estimate signal parameters, a recently proposed quasi maximum-likelihood (QML) estimator is adopted. The proposed algorithm consists of two successive steps: (1) noise influence mitigation by using the proposed normalization strategy and (2) signal parameters estimation using the DOA-QML approach. The algorithm performance is evaluated for both monocomponent and multicomponent signals.
\end{abstract}

K e y w ords: polynomial-phase signal, impulsive noise, Fourier transform, uniform linear array, direction of arrival, parameter estimation

\section{Introduction}

Direction-of-arrival (DOA) estimation is a fundamental problem in numerous applications including radars, sonars, underwater acoustics, indoor navigation and communications $[1-3]$. DOA is commonly estimated from signal captured by array of sensors. Depending on application, there are various array geometries such as uniform linear array (ULA), uniform rectangular array (URA), uniform circular arrays (UCA), co-prime arrays, etc [4-6]. The ULA is the most popular one and it is used to form other geometries. In the literature, the DOA is estimated by using eigenvalues [7-8], meta-heuristic search algorithms [6], phase differentiation [9-13], compressive sensing [14], time-frequency distributions [15], etc.

Dealing with signals corrupted by non-Gaussian noise, in particular impulsive and/or heavy tailed noise, is common in array signal processing [16-24]. Such disturbances can significantly reduce performance of techniques developed for Gaussian noise environment. In many disciplines, the nonlinear signal processing approach is a common tool for reducing negative effects of the impulsive/heavy-tailed noise. For example, in signal and image processing, numerous robust filter families related to modifications and upgrades of the median filter have been developed in last two decades [25-27]. However, robust filters require sorting that is computationally demanding operation. Knowing that sensor nodes are often of limited resources and that processing can introduce some delays in real-time evaluation, their application is limited.

Normalization/regularization and hard/soft limiters implementations are also common tools in robust signal processing and robust statistics [28]. This paper proposes the normalization strategy based approach for estimation of DOA and parameters of signals impinging on the ULA of sensors. Signals received by the ULA are represented by the polynomial phase signal (PPS) model and a recently developed DOA-quasi maximum likelihood (DOAQML) algorithm [15,29] is coupled with the proposed normalization strategy. When Gaussian noise is considered, the DOA-QML algorithm has excellent performance compared to other state-of-the-art estimators [6, 9-12]. Thus, the proposed extension is important for covering challenging noise environments. The algorithm consists of two stages. In the first stage, ensemble of sets of parameter estimates with various normalization factors is produced, while in the second stage, the optimal set is selected based on an appropriate criterion function.

\section{Problem statement}

Consider a source emitting $\mathrm{PPS}^{1}$ defined as $[31,32]$

$$
\begin{aligned}
x(n) & =A \exp (j \phi(n))+\nu(n) \\
& =A \exp \left(j a_{0}+j \sum_{m=1}^{M} a_{m} \frac{n^{m}}{m}\right)+\nu(n),
\end{aligned}
$$

where $A$ is the constant or slowly varying amplitude, $M$ is the signal order, $\left\{a_{m} \mid m \in[0, M]\right\}$ are phase parameters, while $\nu(n)$ is additive noise. Assume that noise is zero-mean complex with independent real and imaginary parts but of impulsive or heavy tailed nature and the number of signal samples is $N$.

${ }^{1}$ PPS signals are motivated by the Weierstrass theorem that states that any continuous function on finite interval can be modeled by polynomial [30].

\footnotetext{
${ }^{1}$ University of Montenegro, Electrical Engineering Department, Cetinjski put bb, 81000 Podgorica, ${ }^{2}$ University of Donja Gorica, Faculty for Information Systems and Technologies, Oktoih 1, 81000 Podgorica, Montenegro, ${ }^{3}$ National Aerospace University, Department of Information-Communication Technologies, 61000 Kharkov, Ukraine, * Corresponding author marko.simeunovic@udg.edu.me
} 
Signal (1) is received by the ULA consisting of $L$ sensors separated with intersensor distance $d$. Since sensor positions differ, the $l$ th sensor will capture delayed version of (1)

$$
x_{l}(n)=x(n-l \tau), \quad l \in[0, L),
$$

where $\tau=d \sin \theta / c, \theta$ is DOA, and $c$ is speed of signal propagation in considered environment. Our goal is to estimate phase parameters $\left\{a_{m} \mid m \in[0, M]\right\}$ and intersensor delay $\tau$ from noisy observations $x(n)$.

Equation (2) represents a general wideband ULA signal model. In practice, it has been commonly assumed that instantaneous frequency (IF), $\omega(n)=\sum_{m=1}^{M} a_{m} n^{m-1}$ does not change during travel of impinging signal across the ULA [6]. In that case, $\tau$ is relatively small, signal is narrowband and $x_{l}(n) \approx A \exp (j \phi(t)-j l \tau \omega(t))$. Narrowband signals can be more easily processed compared with wideband ones. Here we will consider general case and our approach can be adopted for the simplified model.

\section{Algorithm development}

\subsection{Monocomponent signals}

The PPS estimation in the ULA sensor network is popularized by [6] where technique referred to as the chirp/polynomial beamformer $(\mathrm{CB} / \mathrm{PB})$ is proposed. The $\mathrm{PB}$ is the maximum-likelihood (ML) estimator of the DOA and phase parameters of signal (2). It can be described as

$$
\begin{gathered}
J\left(\xi ; \alpha_{1}, \ldots, \alpha_{M}\right)= \\
\sum_{l} \sum_{n}\left|x_{l}(n) \exp \left(-j \sum_{m=1}^{M} \alpha_{m} \frac{(n-l \xi)^{m}}{m}\right)\right|^{2} \\
\left(\hat{\tau} ; \hat{a}_{1}, \ldots, \hat{a}_{M}\right)=\arg \max _{\left(\xi ; \alpha_{1}, \ldots, \alpha_{M}\right)} J\left(\xi ; \alpha_{1}, \ldots, \alpha_{M}\right) .
\end{gathered}
$$

The main drawback of the PB-based approach is $(M+$ 1)-dimensional search that significantly limits its applicability. In [6], in order to avoid the direct search, even for the second order PPS, the PB maximization is performed by the genetic algorithm.

To reduce calculation complexity, the phase differentiation (PD)-based methods are used as alternatives to the PB approach [9-12]. The PD, realized by auto-correlation function, decrements signal order reducing the search dimension at the same time. However, it introduced numerous issues. Some of them are common to the PD estimators applied on a single sensor: increased breakdown point and mean squared error (MSE), error propagation from higher toward lower order phase parameters, problems with cross-terms, etc. The other shortcomings are caused by sensor architecture itself. For example, intersensor delays causing difficulties in fusing results of the PD procedures applied among sensors since particular phase function and observed phase coefficients on sensors are different. Some of techniques addressing these issues are reviewed in [33, 34].

The QML-based estimation procedure has been proposed in order to avoid shortcomings of the PB- and PDbased estimators [33]. This technique can be conceptually described as hybrid of the linear time-frequency (TF) representations, phase unwrapping and ML estimator with advantages drawn from all of them but without associated peculiarities. The QML does not require PDs and unlike $(M+1)$-dimensional search required by the $\mathrm{PB}$, it performs 1-D search only. This technique consists of three main steps:

Step 1 Coarse parameter estimation - signal parameters are estimated using linear TF representation, ie, short time-Fourier transform (STFT);

Step 2 Fine parameter estimation - the O'Shea strategy [35] based on phase unwrapping and signal filtering is used to remove bias caused by the STFT-based estimator and to improve the estimation accuracy close to achievable limits given by the Cramer-Rao lower bounds (CRLB);

Step 3 Selection of optimal estimate - the ML function is used to determine the optimal estimate by searching over set of windows widths in the STFT.

The QML has been applied in numerous important scenaria including: non-Gaussian noise, random samples consensus algorithm (RANSAC), fading amplitude or short signal components, for different parametric signal models but also for unknown signal models, multicomponent signals, multidimensionalsignals, etc [33]. One of the main advantages of the QML algorithm is fact that its accuracy is stable with respect to the phase order increase. It is well known that many of existing phase parameter estimators have performance significantly deteriorating with increase of the phase order [33]. For example, the high-order ambiguity (HAF)-based estimators have decreasing breakdown point of the algorithm for $6 \mathrm{~dB}$ with each order of polynomial in the signal phase. However, the QML is tested on high-order polynomials even for $M=40$ with minor loss of accuracy [33]. In addition, the QML is applied to nonpolynomial modulated signals showing excellent accuracy [33].

Our goal is to exploit usage of the QML in the estimation of parameters of PPS captured by the ULA in impulsive/heavy-tailed noise environments. This problem has been already addressed in [33] where the robust QML is proposed. In its initially stage, the algorithm calculates the robust TF representation instead of the STFT. The robust filtering in time domain is used in refinement stage and selection of optimal estimate is performed with criterion function appropriate for non-Gaussian environment. However, this approach is computationally complex since the robust TF representations require time consuming sorting operations for each point in the TF plane. In array signal processing applications, such calculation complexity is unacceptable due to weak computation capabilities of sensor nodes and long signal recordings. 
To reduce influence of impulsive noise and improve both calculation complexity and estimation accuracy, in this paper, we propose signal pre-processing prior to parameter estimation using the classical DOA-QML algorithm [15]. Signal can be pre-processed by utilization of the following normalization strategy [28]

$$
x_{l, b}(n)=\frac{x_{l}(n)}{\sqrt{\left|x_{l}(n)\right|^{2}+b^{2}}},
$$

where $b$ is factor that has influence on normalization characteristics. For $b \rightarrow \infty$ relation (4) has output proportional to the input signal without reducing effects of impulses, while for $b \rightarrow 0$ the fractional lower order normalized signal [36] is obtained. Distortion of signal content is a side effect of the normalization procedure. Therefore, parameter $b$ should be selected as a trade-off between impulsive noise suppression and signal distortion.

Algorithm 1: Robust DOA-QML estimation

Input: Signal $x_{l}(n), l \in[0, L)$ and set of intersensor delays $\mathbf{T}$

First stage: Estimation of normalization parameter $b$

Perform signal normalization with $b=0$

$$
x_{l, 0}(n)=\frac{x_{l}(n)}{\left|x_{l}(n)\right|} .
$$

Using the DOA-QML algorithm, estimate signal parameters

$$
\left\{\hat{a}_{1}^{0}, \ldots, \hat{a}_{M}^{0} ; \hat{\tau}^{0}\right\}=\operatorname{DOA}-\mathrm{QML}\left\{x_{l, 0}(n)\right\} .
$$

Estimate the signal amplitude as

$$
\hat{A}=\sqrt{\begin{array}{l}
{\left[\frac{1}{L}\left|\operatorname{median}\left\{\operatorname{Re}\left[\sum_{l=0}^{L-1} x_{l}(n) \exp \left(-j \sum_{m=1}^{M} \hat{a}_{m}^{0} \frac{\left(n-l \hat{\tau}^{0}\right)^{m}}{m}\right)\right]\right\}\right|^{2}\right.} \\
+\frac{1}{L}\left|\operatorname{median}\left\{\operatorname{Im}\left[\sum_{l=0}^{L-1} x_{l}(n) \exp \left(-j \sum_{m=1}^{M} \hat{a}_{m}^{0} \frac{\left(n-l \hat{\tau}^{0}\right)^{m}}{m}\right)\right]\right\}\right|^{2}
\end{array}}
$$

Second stage: Re-estimation of signal parameters

Based on the estimated amplitude, update $b$ parameter as

$$
b=\zeta \hat{A},
$$

where (as it will be demonstrated in numerical experiments) accurate results are obtained for wide range of parameter $\zeta$ (we adopted $\zeta \in[0.3,0.6]$ what will be demonstrated in simulation section). Re-estimate signal parameters using the DOA-QML algorithm as

$$
\left\{\hat{a}_{0}^{b}, \hat{a}_{1}^{b}, \ldots, \hat{a}_{M}^{b} ; \hat{\tau}^{b}\right\}=\operatorname{DOA}-\mathrm{QML}\left\{x_{l, b}(n)\right\},
$$

where signal is normalized with parameter $b$ from

$$
x_{l, b}(n)=\frac{x_{l}(n)}{\sqrt{\left|x_{l}(n)\right|^{2}+b^{2}}} .
$$

Output: Signal parameters estimate $\left\{\hat{a}_{0}, \hat{a}_{1}, \ldots, \hat{a}_{M} ; \hat{\tau}\right\}=\left\{\hat{a}_{0}^{b}, \hat{a}_{1}^{b}, \ldots, \hat{a}_{M}^{b} ; \hat{\tau}^{b}\right\}$

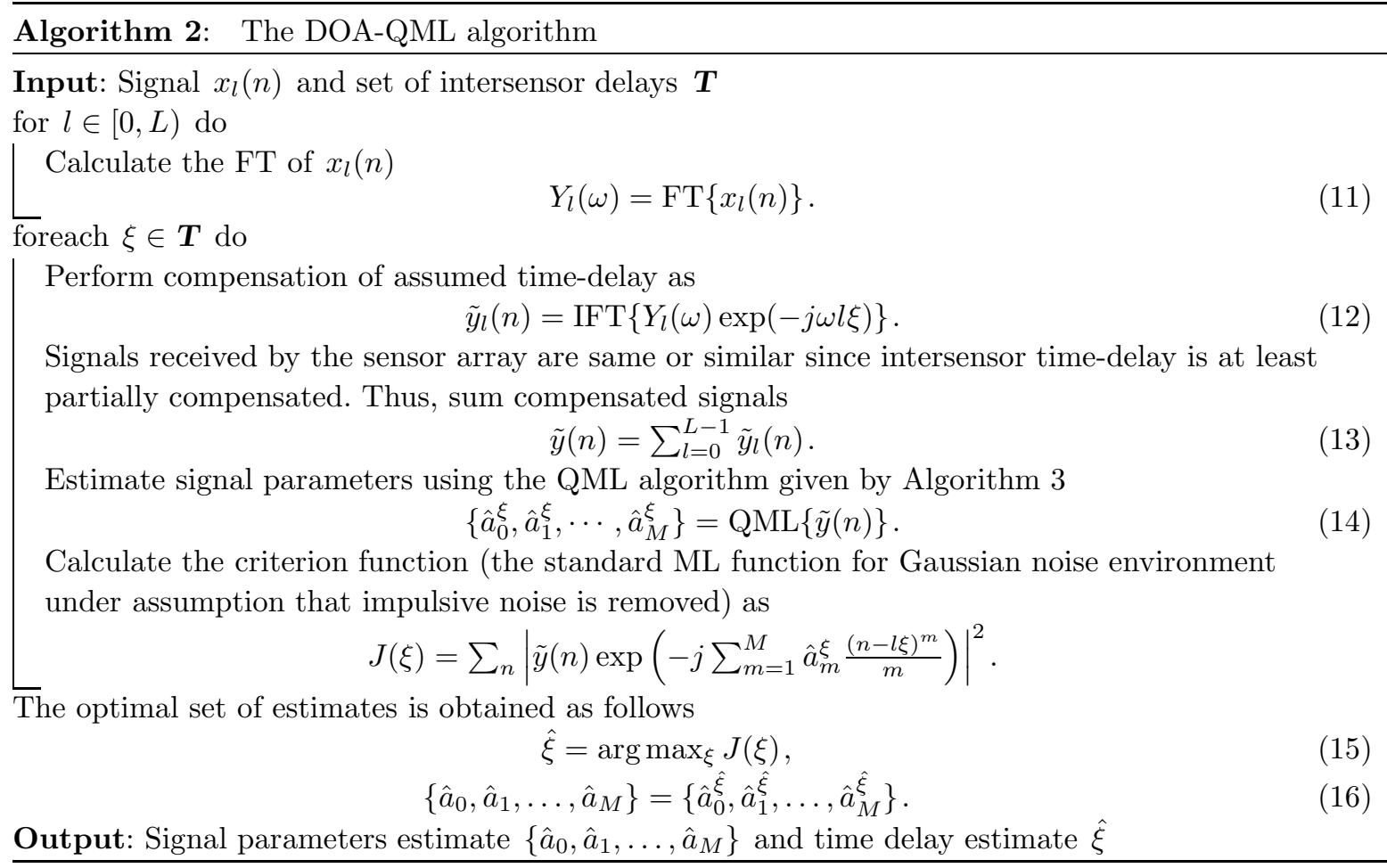




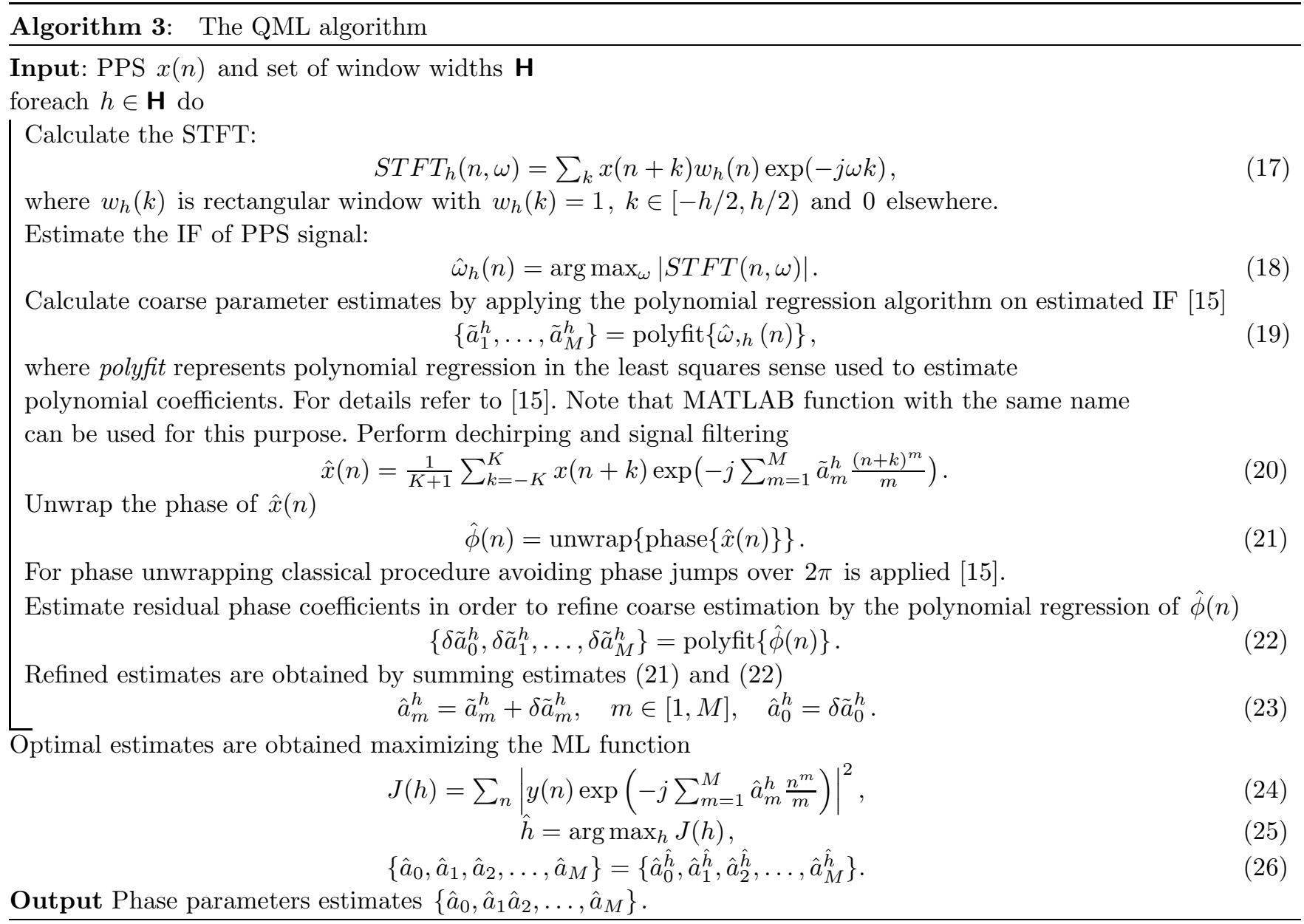

Algorithm 4: The DOA-QML algorithm for multicomponent signals

Input: Multicomponent signal $x_{l}(n), l \in[0, L)$ with $Q$ components having parameters

Perform signal normalization with $b=0$

$$
\left\{a_{q 0}, a_{q 1}, \ldots, a_{q M} ; \tau_{q}, A_{q}\right\} \text {. }
$$

$$
x_{l, 0}(n)=\frac{x_{l}(n)}{\left|x_{l}(n)\right|}
$$

Estimate parameters of the strongest component $(q=1)\left\{\hat{a}_{11}^{0}, \ldots, \hat{a}_{1 M}^{0} ; \hat{\tau}_{1}^{0}\right\}$ and perform signal dechirping

Calculate the FT of signal $(28), \hat{X}_{l}(\omega)=\operatorname{FT}\left\{x_{l}(n)\right\}$.

$$
\hat{x}_{l}(n)=x_{l}(n) \exp \left(-j \sum_{m=1}^{M} \hat{a}_{1 m}^{0} \frac{\left(n-l \hat{\tau}_{1}^{0}\right)^{m}}{m}\right) \text {. }
$$

Remove components that are away from the origin

$$
\hat{X}_{l}(\omega)=0 \text { for }|\omega| \geq|\Delta \omega| .
$$

Perform evaluation of the inverse FT, $\tilde{x}_{l}(n)=\operatorname{IFT}\left\{\hat{X}_{l}(\omega)\right\}$, and estimate amplitude of the strongest component as

$$
\hat{A}_{1}=\sqrt{\begin{array}{l}
\frac{1}{L}\left|\operatorname{median}\left\{\operatorname{Re}\left[\sum_{l=0}^{L-1} \tilde{x}_{l}(n) \exp \left(-j \sum_{m=1}^{M} \hat{a}_{1 m}^{0} \frac{\left(n-l \hat{\tau}_{1}^{0}\right)^{m}}{m}\right)\right]\right\}\right|^{2} \\
+\frac{1}{L}\left|\operatorname{median}\left\{\operatorname{Im}\left[\sum_{l=0}^{L-1} \tilde{x}_{l}(n) \exp \left(-j \sum_{m=1}^{M} \hat{a}_{1 m}^{0} \frac{\left(n-l \hat{\tau}_{1}^{0}\right)^{m}}{m}\right)\right]\right\}\right|^{2}
\end{array}}
$$

Estimate normalization parameter $\hat{b} \in \zeta \hat{A}_{1}$. Then we can proceed with re-estimation of the strongest component and estimation of all other components using normalization parameter $\hat{b}$ as follows.

for $q=0$ to $Q$ do

Estimate parameters of $q$ th component $\left\{\hat{a}_{q 1}^{\hat{b}}, \ldots, \hat{a}_{q M}^{\hat{b}} ; \hat{\tau}_{q}^{\hat{b}}\right\}$ using the DOA-QML algorithm.

Demodulate received signal with estimated components parameters

Eliminate DC component of $\hat{x}_{l}(n)$

$$
\hat{x}_{l}(n)=x_{l}(n) \exp \left(-j \sum_{m=1}^{M} \hat{a}_{q m}^{\hat{b}} \frac{\left(n-l \hat{\tau}_{q}^{\hat{b}}\right)^{m}}{m}\right) .
$$

$$
\begin{aligned}
& \hat{Y}_{l}(\omega)=\operatorname{FT}\left\{\hat{x}_{l}(n)\right\}, \\
& \hat{Y}_{l}(\omega)=0, \\
& \hat{x}_{l}(n)=\operatorname{IFT}\left\{\hat{Y}_{l}(\omega)\right\} . \\
& x_{l}(n)=\hat{x}_{l}(n) \exp \left(j \sum_{m=1}^{M} \hat{a}_{q m}^{\hat{b}} \frac{\left(n-l \hat{\tau}_{q}^{\hat{b}}\right)^{m}}{m}\right) .
\end{aligned}
$$

Modulate signal

Output: Final estimates $\left\{\hat{a}_{q 1}, \ldots, \hat{a}_{q M} ; \hat{\tau}_{q}\right\}, q=1, \ldots, Q$ 
Some other normalization strategies used in robust signal processing are reviewed in [37-39].

The classical approach in the selection would consider brute force search for optimal $b$ in some predefined interval. This would lead to significant increase of calculation complexity. In order to keep complexity as low as possible, we propose to perform selection/estimation through two-stage procedure:

Stage 1 Set $b=0$, perform normalization and estimate signal amplitude $A$ using the DOA-QML approach;

Stage 2 Normalize signal $x_{l}(n)$ by $b$ selected to be proportional to amplitude obtained in Stage 1 and estimate its parameters and intersensor delay by the DOA-QML.

Normalization influences primarily amplitude of the signal that can be affected by impulsive noise. The DOAQML in its course estimation stage estimates signal parameters by estimating IF using the STFT. The IF is inversely proportional to number of zero-crossings $[40,41]$ that is not affected by changes in the signal amplitude. Therefore, normalization would have minimal influence to IF estimation while at the same time reduces effect of impulsive noise.

The basic structure of the robust DOA-QML algorithm for monocomponent signals described in (2) is given by Algorithm 1.

The DOA-QML algorithm used in (9) is summarized by Algorithm 2. Firstly, for predefined set of possible inter sensor delays, the method performs time-delay compensation of signal received by each sensor using the FFT. Since their arrival times are now synchronized, compensated recordings are summed to form vector whose parameters are estimated by the QML algorithm developed for single sensor case (Algorithm 3, already explained in Introduction). In this way, several sets of parameter estimates are obtained. Optimal one corresponds to $\tau$ that maximizes criterion function.

\section{Calculation complexity}

The proposed algorithm calculates the DOA-QML approach twice and this step is dominant operation that determines the overall complexity of the estimation procedure. Calculation complexity of the DOA-QML has been already derived in [15] and it is of order of $\mathrm{O}\left(\|H\| N^{2}|| \Omega|| \log _{2} N\right)$ operations, where $\boldsymbol{H}$ is set of window width used for the QML algorithm evaluation, $\boldsymbol{T}$ set of intersensor delays, $N$ number of signal samples and $\|\cdot\|$ operator determines number of elements in the corresponding set. If the STFT is realized recursively, this can be reduced to $\mathrm{O}\left(\|H\| N^{2}\|\Omega\|\right)$ operations [42].

In order to illustrate benefits of signal normalization strategy (4) consider the robust variant of the QML. The robust DOA-QML algorithm evaluated by marginal median STFT would require $\mathrm{O}\left(\|H\| \cdot\|\Omega\| N^{3} \log _{2} 2 N\right)$ operations that is significantly larger comparing to the complexity of the proposed approach [33].

\subsection{Multicomponent signals}

When multicomponent signals are considered, there are several potential strategies for the selection of normalization parameter $b$. Similar strategy to monocomponent case can be adopted here. Thus, one have firstly to perform estimation of phase signal parameters and amplitudes by considering $b=0$ and in the next step to perform re-estimation by considering signal where each component is normalized according to optimal $b$ value.

An alternative to this approach is to estimate amplitude of the strongest component only, and to perform re-estimation of normalized signal where each component is normalized using $b$ calculated based on estimated amplitude. It is more efficient approach with respect to potential adjustment of $b$ for each component not affecting significantly accuracy of the proposed technique. The algorithm is summarized by Algorithm 4.

\section{Numerical examples}

In this section, performance of the proposed approach is compared with classical DOA-QML- and HAF-based algorithms from [9] and [11]. Experiments are performed considering signals corrupted by $\alpha$-stable noise and mixed Gaussian and impulsive noise on both monocomponent and multicomponent signals.

To avoid misunderstanding, we introduce the following notations:

- DOA-QML-C - the DOA-QML approach applied on original signal (classical DOA-QML);

- DOA-QML-N - the DOA-QML approach applied on normalized signal (proposed approach);

- HAF - the HAF-based approach from [11] applied on original signal;

- HAF-N - the HAF-based approach from [11] applied on normalized signal;

- AHAF-N - the HAF-based approach from [9] applied on normalized signal.

\section{Monocomponent signal corrupted by $\alpha$-stable noise}

Consider the ULA consisting of $L=25$ sensors separated with $d=0.5 \mathrm{~m}$. The third-order PPS with parameters $A=1, a_{0}=\pi / 3, a_{1}=21 / N, a_{2}=34 / N^{2}$ and $a_{3}=36 / N^{3}$ is captured by the ULA at angle $\theta=\pi / 8$. Number of samples and sampling interval are respectively $N=256$ and $\Delta t=1$. A widely used model of nonGaussian (heavy-tailed) noise is the $\alpha$-stable noise characterized by two basic parameters $\alpha$ (responsible for impulsivity) and $\gamma$ (that deals with noise intensity) $[37,43]$. The noise parameters are unknown in advance and their estimation is very difficult. Thus, we analyze a wide range of noise impulsivity to show that the proposed method is robust enough in wide sense. Signal is corrupted by the $\alpha$-stable noise with parameters $\alpha \in[0.8,2]$ and $\gamma=0.2$. 

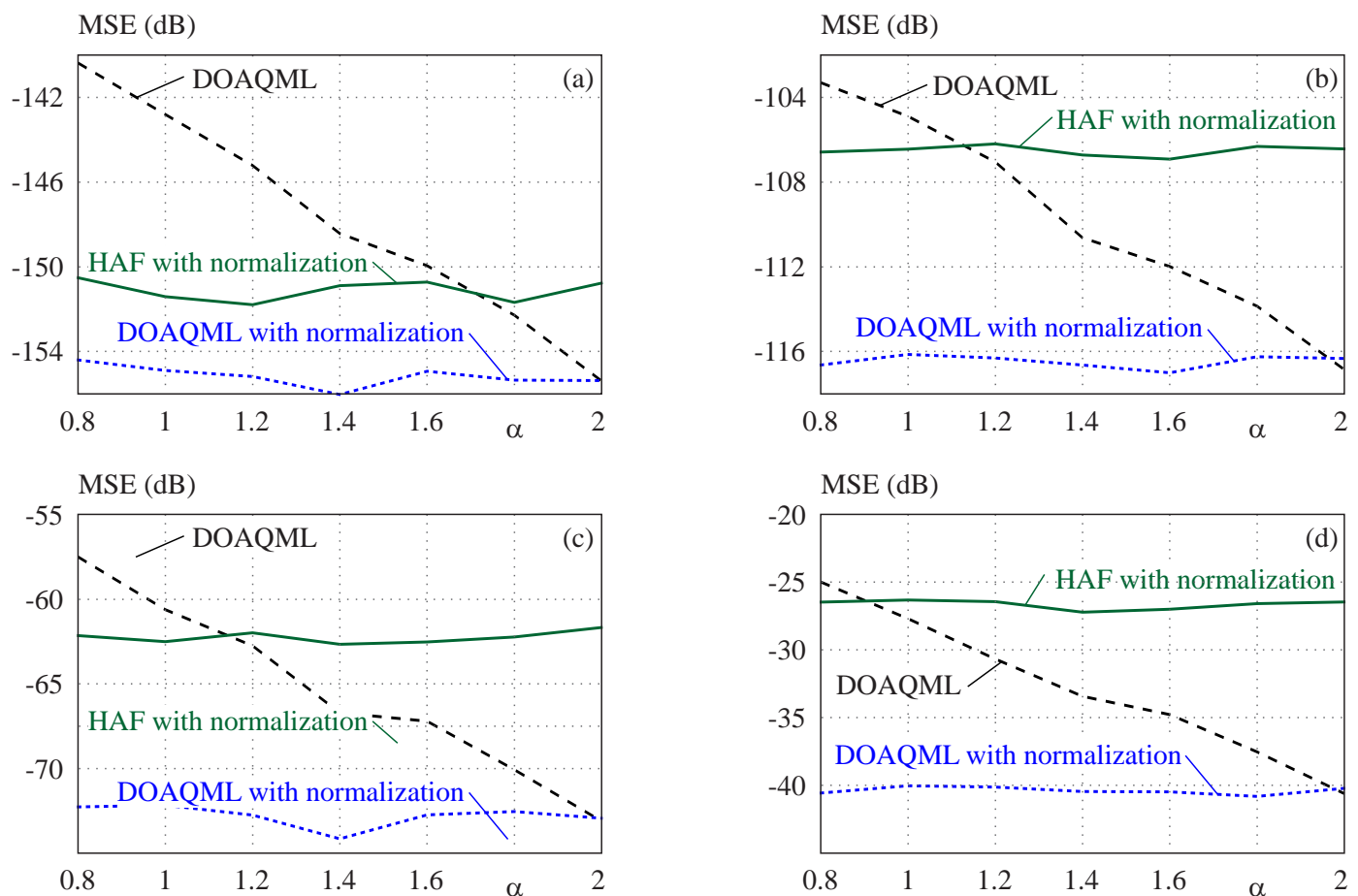

Fig. 1. MSE of phase parameters: $\left(a_{3}, a_{2}, a_{1}\right)$ and intersensor delay $(\tau)$ estimates for signal corrupted by $\alpha$-stable noise impinging on the ULA obtained by the DOA-QML-C, DOA-QML-N, HAF-N and AHAF-N

$\operatorname{MSE}(\mathrm{dB})$

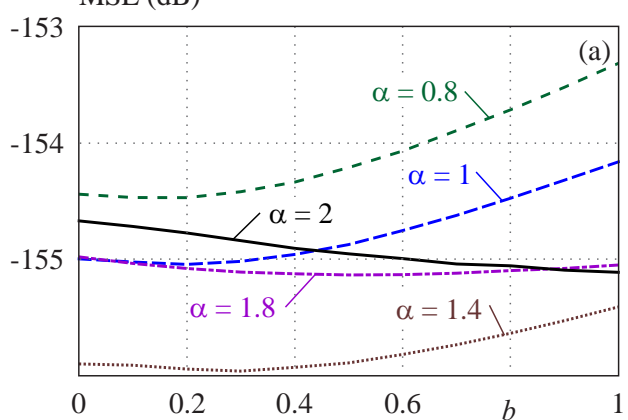

$\operatorname{MSE}(\mathrm{dB})$

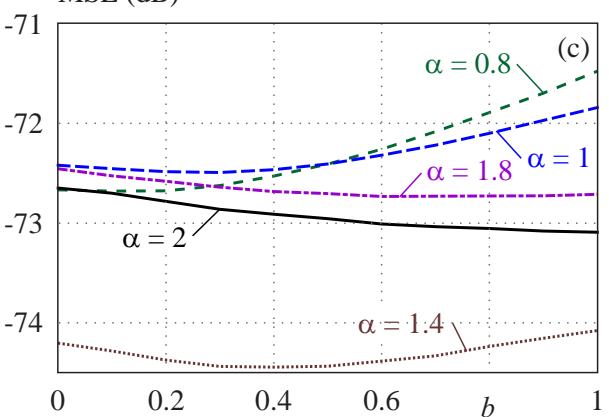

$\operatorname{MSE}(\mathrm{dB})$

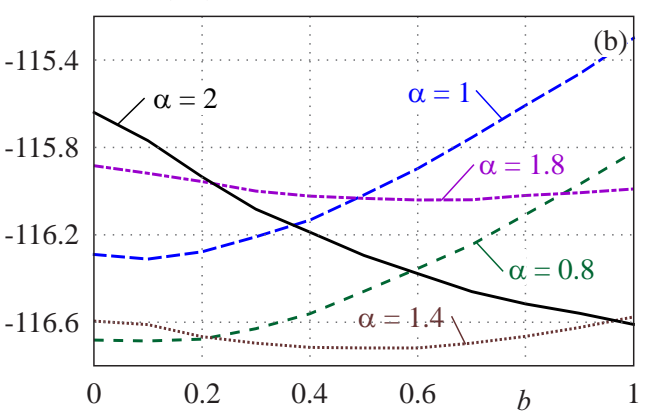

$\operatorname{MSE}(\mathrm{dB})$

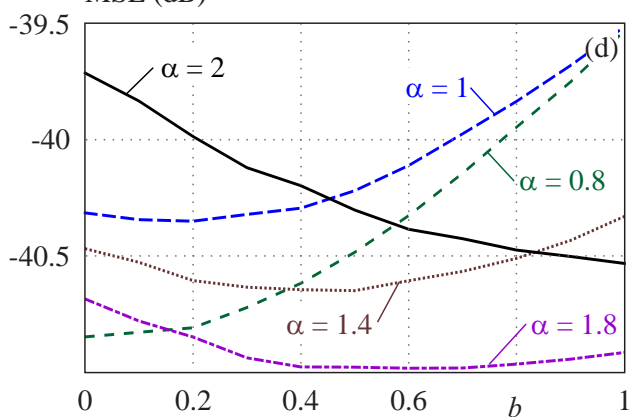

Fig. 2. MSEs of phase parameters and intersensor delay estimates for $\alpha$-stable noise and DOA-QML-N as function of normalization factor $b$

Parameter $\alpha$ corresponds to tail of the probability density function, ie, number of impulses in the signal. For example, $\alpha=1$ represents the Cauchy noise with infinite variance. Smaller $\alpha$ corresponds to the heavier impulsive noise contribution while for $\alpha=2$ the Gaussian noise with variance proportional to $\gamma$ is obtained.
The MSEs of phase parameters $\left\{a_{3}, a_{2}, a_{1}\right\}$ and intersensor delay $\tau$ obtained by the DOA-QML-C, DOAQML-N, HAF-N and AHAF-N are given in Fig. 1. MSEs are calculated using 200 trials of Monte Carlo simulations while signal normalization is done considering $\zeta=0.5$. Obtained results clearly demonstrate advantages of the 


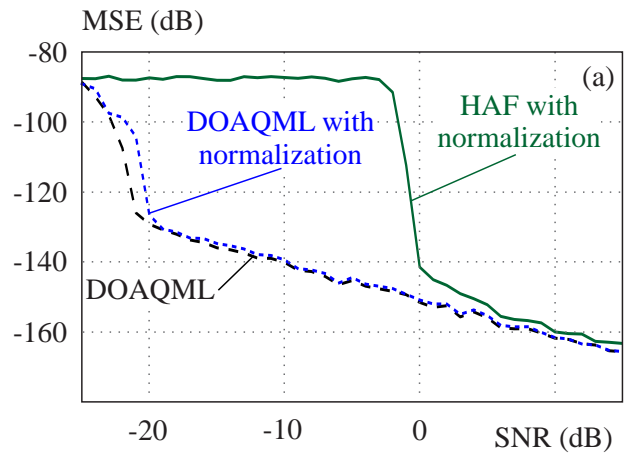

$\operatorname{MSE}(\mathrm{dB})$

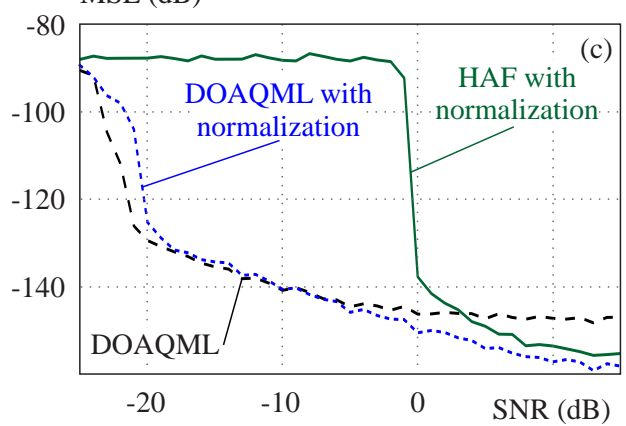

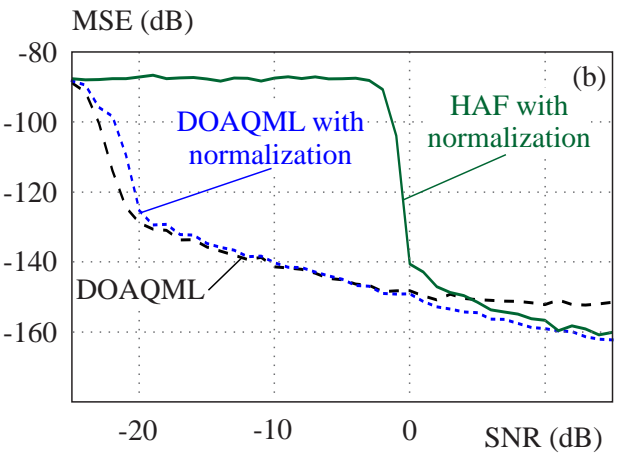

$\operatorname{MSE}(\mathrm{dB})$

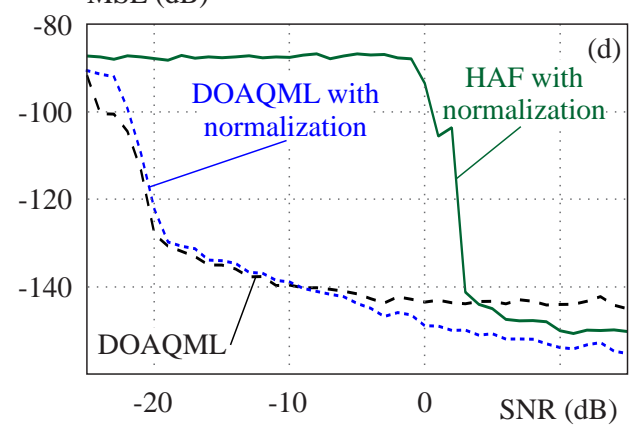

Fig. 3. Estimation of parameter $a_{3}$ for mixed Gaussian and impulsive noise environment for $3 \%, 10 \%, 20 \%$ and $30 \%$ of impulses as function of the SNR (ratio between signal ampitude and Gaussian noise variance)

Table 1. MSEs of two component signal impinging on the ULA corrupted by Cauchy noise obtained by the DOA-QML-N and DOAQML-C algorithms

\begin{tabular}{lcccccccc}
\hline & \multicolumn{3}{c}{ First component } & \multicolumn{3}{c}{ Second component } \\
\hline MSE(dB) & $\hat{a}_{3}$ & $\hat{a}_{2}$ & $\hat{a}_{1}$ & $\hat{\tau}$ & $\hat{a}_{3}$ & $\hat{a}_{2}$ & $\hat{a}_{1}$ & $\hat{\tau}$ \\
DOA-QML-C & -142.81 & -97.08 & -60.34 & -37.49 & -139.55 & -103.56 & -57.70 & -38.29 \\
DOA-QML-N & -153.93 & -97.36 & -70.57 & -45.87 & -148.64 & -111.91 & -67.18 & -48.71 \\
\hline
\end{tabular}

proposed approach over counterparts. The MSE of the DOA-QML-C (case when normalization is not performed) significantly increases as amount of impulsiveness increases (with reducing $\alpha$ ) while the proposed technique (DOA-QML-N) gives rather stable results outperforming other considered techniques (the HAF-N, AHAF-N and DOA-QML-C). For example, for the Cauchy noise ( $\alpha=1$ ), the proposed normalization approach has lower MSE with respect to the DOA-QML-C for more than $12 \mathrm{~dB}$ while for other phase parameters, improvement is even higher and it is up to $14 \mathrm{~dB}$. It can be seen that MSEs of normalization based approaches (DOA-QML$\mathrm{N}, \mathrm{HAF}-\mathrm{N}$ and AHAF-N) are approximately parallel, ie normalization keeps them on similar level not influenced by impulsive noise strength. However, the DOAQML-N outperforms the HAF-N and AHAF-N counterparts between $4 \mathrm{~dB}$ and $14 \mathrm{~dB}$ depending on the considered parameter. The DOA-QML-C, as expected, outperforms the DOA-QML-N only for $\alpha=2$ (Gaussian noise). This improvement is less than $0.2 \mathrm{~dB}$ for all parameters.
In order to justify selection of parameter $b$, in Fig. 2, we show MSEs obtained in the estimation of phase parameters $\left\{a_{3}, a_{2}, a_{1}\right\}$ and intersensor delay $\tau$ by considering several values of normalization parameter $b$. From Fig. 2, it can be seen that MSE variations with respect to parameter $b$ are less than $1 \mathrm{~dB}$ for all $\alpha$ confirming our decision to take estimate of $b$ as $b=\zeta \hat{A}$, where $\zeta \in[0.3,0.6]$.

\section{Monocomponent signal with mixed Gaussian and impulsive noise}

Signal from previous example is corrupted by mixed Gaussian and impulsive noise. Impulses positions are randomly selected and their magnitudes are set to be five times larger that the signal amplitude. Half of impulses had positive, while remaining half had negative values. Percentage of impulses in signal is controlled with parameter $p$ that took the following values $3 \%, 10 \%$, $20 \%$, and $30 \%$. The variance of the Gaussian noise component is $\sigma^{2}$ and signal-to-noise ratio (SNR) is calcu- 

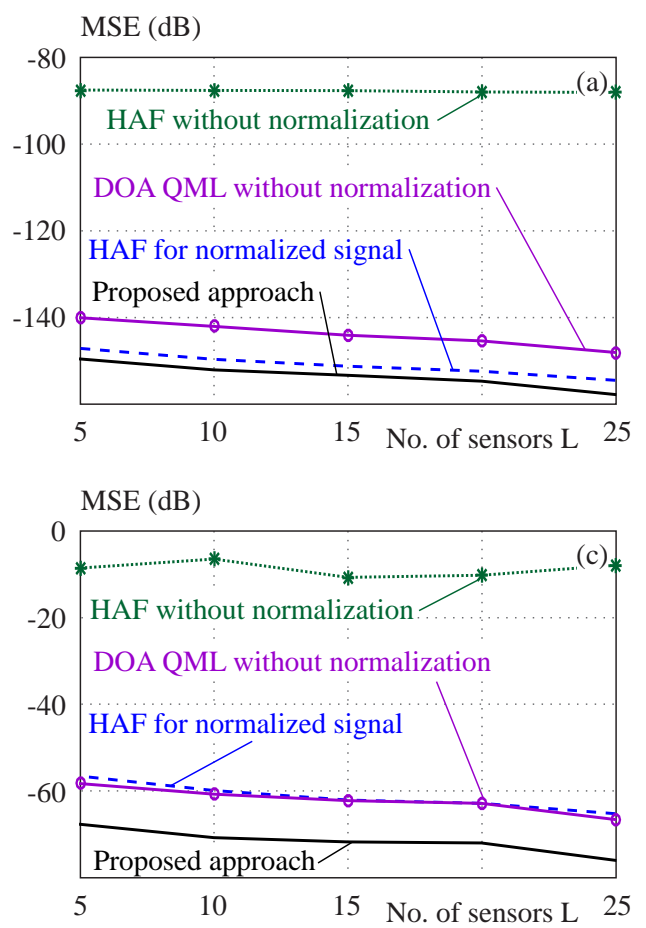

$\operatorname{MSE}(\mathrm{dB})$

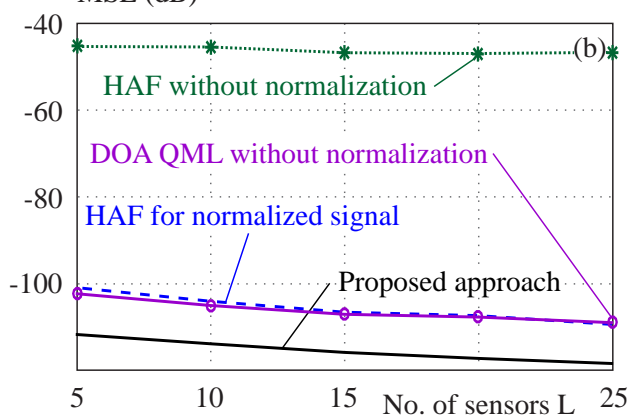

$\operatorname{MSE}(\mathrm{dB})$

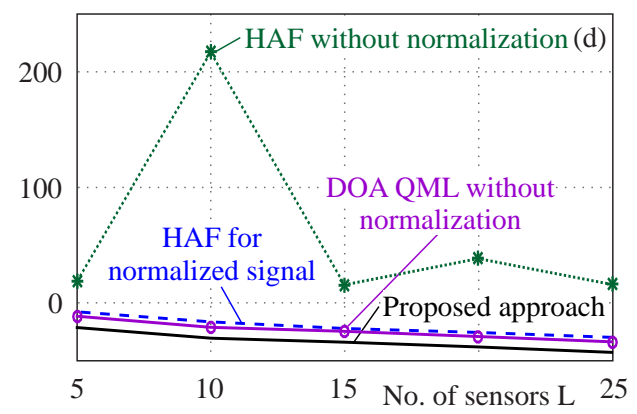

Fig. 4. MSEs of $a_{3}, a_{2}, a_{1}$ and $A$ parameter estimates as functions of sensors numbers obtained by the DOA-QML-C, DOA-QML-N, HAF-C and HAF-N

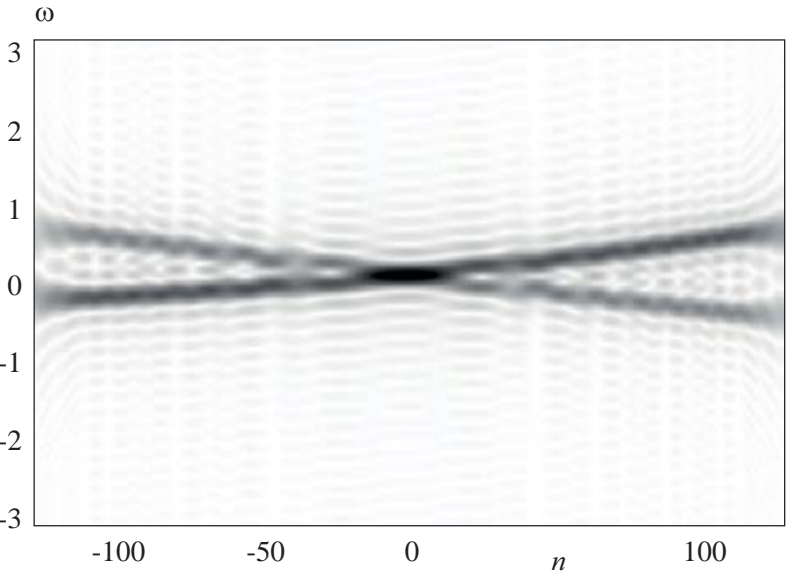

Fig. 5. STFT of two-component PPS signal considered in numerical examples.

lated with respect to Gaussian noise component only, ie $\mathrm{SNR}=10 \log 10\left[A^{2} / \sigma^{2}\right]$. MSEs of the DOA-QMLC, DOA-QML-N, HAF-N and AHAF-N $a_{3}$ estimates obtained by 250 Monte Carlo simulations are shown in Fig. 3.

As expected, for small percentage of impulses, the DOA-QML-C and the DOA-QML-N have similar behavior. However, with increase of percentage of impulsive noise, we are witnessing advantage of the proposed technique. For example, for $p=10 \%$ and $\mathrm{SNR}=15 \mathrm{~dB}$, improvement with the proposed strategy is more than $11 \mathrm{~dB}$ and improvement is less emphatic as Gaussian noise component increases. Further increase of $p$ does not influence the proposed technique and again improvement with respect to the DOA-QML-C is increasing. For example, for $p=20 \%$ and $\mathrm{SNR}=5 \mathrm{~dB}$, improvement is about $7 \mathrm{~dB}$.

Figure 4 depicts influence of the number of sensors $L$ to the accuracy of the proposed technique in comparison with DOA-QML-C, HAF-C and HAF-N. Noise parameters are $\mathrm{SNR}=10 \mathrm{~dB}$ and $p=10 \%$. The proposed technique outperforms all alternatives for at least $2 \mathrm{~dB}$. The HAF-C-based technique is far the worst and not useful for the impulsive noise case. It is important to note that the proposed technique is suitable even for relatively small number of sensors $L=5$.

\section{Multicomponent signal}

The proposed algorithm has been tested on the thirdorder two-component signal corrupted by the Cauchy noise $(\alpha=1$ and $\gamma=0.2)$. Signal parameters are:

first component - amplitude $A_{1}=1$, DOA $\theta_{1}=\pi / 3$, phase parameters $a_{0,1}=\pi / 3, a_{1,1}=21 / N, a_{2,1}=$ $120 / N^{2}$ and $a_{3,1}=48 / N^{3}$

second component - amplitude $A_{2}=0.7$, DOA $\theta_{2}=$ $\pi / 12$ and phase parameters $a_{0,1}=\pi / 4, a_{1,1}=16 / N$, $a_{2,1}=-150 / N^{2}$ and $a_{3,1}=12 / N^{3}$.

The STFT of signal captured by the first sensor is given in Fig. 5. From figure, one can notice that signal components are overlapping in the $\mathrm{TF}$ plane. For many techniques, parameter estimation of this signal is very challenging. However, the proposed approach is able to accurately estimate signal parameters as far as their magnitudes differs. The estimation procedure can be done by 
estimating component-by-component meaning that the stronger component is first estimated. Then it is peeled from the mixture and the weaker component is estimated. In the case of more complicated signatures, $i e$, overlapping components of the same magnitude, some of components tracking or signal decomposition techniques in the TF plane should be employed.

To obtain MSEs of DOA-QML-C and DOA-QML-N parameter estimates, 250 Monte Carlo simulations are used. Their results are given in Tab. 1. It can be seen that achieved improvement is about $10 \mathrm{~dB}$ with respect to the case without normalization.

\section{Conclusion}

In this paper, the DOA-QML algorithm is extended for heavy tailed noise environments. The proposed algorithm consists of two steps. In the first step, impulsive noise is mitigated by using signal normalization strategy, while signal parameters and DOA are estimated in the second step using the classical DOA-QML algorithm. Experiments performed on both monocomponent and multicomponent signals have shown excellent performance of the proposed approach.

\section{REFERENCES}

[1] J. E. Ball, and N. H. Younan, "Radar and Radio Signal Processing", Multidisciplinary Digital Publishing Institute, 2017.

[2] H. S. Maghdid, I. A. Lami, K. Z. Ghafoor, and J. Lloret, "Seamless Outdoors-Indoors Localization Solutions on Smartphones: Implementation and Challenges", ACM Computing Surveys (CSUR), vol. 48, no. 4, pp. 1-34, 2016.

[3] M. Garcia, J. Tomas, F. Boronat, and J. Lloret, "The development of two systems for indoor wireless sensors self-location", Ad Hoc \& Sensor Wireless Networks, vol. 8, no. 3-4, pp. 235-258, 2009.

[4] H. Xu, Y. Zhang, B. Ba, D. Wang, and P. Han, "Fast TwoDimensional Direction-of-Arrival Estimation of Multiple Signals in Coprime Planar Array", Jornal of Sensors, 2019.

[5] W. Zhang, X. Chen, K. Cui, T. Xie, and N. Yuan, "DOA Estimation for Coprime Linear Array Based on MI-ESPRIT and Lookup Table", Sensors, vol. 18, no. 9, pp. 3043, 2018.

[6] A. B. Gershman, M. Pesavento, and M. G. Amin, "Estimating parameters of multiple wideband polynomial-phase sources in sensor arrays,", IEEE Transactions on Signal Processing, vol. 49, no. 12 , pp. $2924-2934,2001$.

[7] R. Schmidt, "Multiple emitter location and signal parameter estimation", IEEE transactions on antennas and propagation, vol. 34 , no. 3 , pp. 276-280, 1986.

[8] R. Roy, A. Paulraj, and T. Kailath, "Esprit - a subspace rotation approach to estimation of parameters of cisoids in noise", IEEE transactions on acoustics, speech, and signal processing, vol. 34, no. 5 , pp. $1340-1342,1986$.

[9] P. Raković, M. Simeunović, and I. Djurović, "On improvement of joint estimation of DOA and PPS coefficients impinging on ULA", Signal Processing, vol. 134, pp. 209-213, 2017.

[10] A. Amar, "Efficient estimation of a narrow-band polynomial phase signal impinging on a sensor array", IEEE Transactions on Signal Processing, vol. 58, no. 2, pp. 923-927, 2010.

[11] I. Djurović, S. Djukanović, M. Simeunović, P. Raković, and B. Barkat, "An efficient joint estimation of wideband polynomial-phase signal parameters and direction-of-arrival in sensor array", EURASIP Source on Advances in Signal Processing, vol. 2012, no. 1, pp. 1-10, 2012.

12] S. Djukanović, M. Simeunović, and I. Djurović, "Efficient parameter estimation of polynomial-phase signals impinging on a sensor array" Mediterranean Conference on Embedded Computing (MECO), pp. 116-119, 2012.

13] S. Lie, A. Leyman, and Y. Chew, "Wideband chirp parameter estimation in sensor arrays through DPT", Electronics letters, vol. 39, no. 23, pp. 1633, 2003.

[14] S. Qin, Y. D. Zhang, M. G. Amin, and B. Himed, "DOA estimation exploiting a uniform linear array with multiple co-prime frequencies", Signal Processing, vol. 130, pp. 37-46, 2017.

[15] I. Djurović, M. Simeunović, and P. Raković, "Quasi-maximumlikelihood estimator of PPS on the uniform linear array", Circuits, Systems, and Signal Processing, vol. 38, no. 10, pp. 48744889, 2019

[16] L. R. G. Carrillo, W. J. Russell, J. P. Hespanha, and G. E. Collins, "State estimation of multiagent systems under impulsive noise and disturbances", IEEE Transactions on Control Systems Technology, vol. 23, no. 1, pp. 13-26, 2015

17] P. Tsakalides, R. Raspanti, and C. L. Nikias, "Angle/Doppler estimation in heavy-tailed clutter backgrounds", IEEE Transactions on Aerospace and Electronic Systems, vol. 35, no. 2, pp. 419-436, 1999.

18] L. Li, T. Qiu, and X. Shi, "Parameter estimation based on fractional power spectrum density in bistatic MIMO radar system under impulsive noise environment", Circuits, Systems, and Signal Processing, vol. 35, no. 9, pp. 3266-3283, 2016.

[19] L. Li, T.-S. Qiu, and D.-R. Song, "Parameter estimation based on fractional power spectrum under alpha-stable distribution noise environment in wideband bistatic MIMO radar system", AEU-International Source of Electronics and Communications, vol. 67 , no. 11, pp. 947-954, 2013.

20] H. Zhang, and Z. Zheng, "Robust DOA Estimator Under Non-Gaussian Noise and Insufficient Sample Support", Circuits, Systems, and Signal Processing, pp. 1-10, 2020.

[21] Y. Yardimci, A. E. Cetin, and J. A. Cadzow, "Robust direction-of-arrival estimation in non-Gaussian noise", IEEE Transactions on Signal Processing, vol. 46, no. 5, pp. 1443-1451, 1998.

[22] M. Ahmadi, E. Yazdian, and A. A. Tadaion, "An improved G-MUSIC algorithm for non-Gaussian noise condition direction-of-arrival estimation", 23rd Iranian Conference on Electrical Engineering, IEEE, pp. 472-477, 2015.

[23] N. Pramod, and G. Anand, "Nonlinear wavelet denoising for DOA estimation by music", International Conference on Signal Processing and Communications, SPCOM'04, pp. 388-392, 2004.

[24] H. Abeida, and J. P. Delmas, "Robustness of subspace-based algorithms with respect to the distribution of the noise: Application to DOA estimation", Signal Processing, vol. 164, pp. 313-319, 2019.

[25] I. Djurović, and V. V. Lukin, "Robust DFT with high breakdown point for complex-valued impulse noise environment", IEEE Signal Processing Letters, vol. 13, no. 1, pp. 25-28, 2006.

26] J. Astola, and P. Kuosmanen, Fundamentals of nonlinear digital filtering vol. 8, CRC press, 1997

[27] A. Toledo-Lopez, F. J. Gallegos-Funes, and V. Ponomaryov, "Vector median M-type L filter to process multichannel images," Iberoamerican Congress on Pattern Recognition, pp. 54-61, Springer 2008

[28] I. Djurović, LJ. Stanković, and M. Simeunović "Robust time-frequency representation based on the signal normalization and concentration measures", Signal Processing, vol. 104, pp. 424-431, 2014.

29] I. Djurović, and LJ. Stanković, "STFT-based estimator of polynomial phase signals", Signal Processing, vol. 92, no. 11, pp. $2769-2774,2012$. 
[30] N. E. Cotter, "The Stone-Weierstrass theorem and its application to neural networks", IEEE Transactions on Neural Networks, vol. 1, no. 4, pp. 290-295, 1990.

[31] I. Djurović, M. Simeunović, S. Djukanović, and P. Wang, "A hybrid CPF-HAF estimation of polynomial-phase signals: Detailed statistical analysis", IEEE Transactions on Signal Processing, vol. 60, no. 10, pp. 5010-5023, 2012.

[32] Y. Wu, H. C. So, and H. Liu, "Subspace-based algorithm for parameter estimation of polynomial phase signals", IEEE Transactions on Signal Processing, vol. 56, no. 10, pp. 4977-4983, 2008.

[33] I. Djurović, and M. Simeunović, "Review of the quasi-maximum likelihood estimator for polynomial phase signals", Digital Signal Processing, vol. 72, pp. 59-74, 2018.

[34] I. Djurović, M. Simeunović, and B. Lutovac, "Are genetic algorithms useful for the parameter estimation of FM signals?", Digital Signal Processing, vol. 22, no. 6, pp. 1137-1144, 2012.

[35] P. O'Shea, "On refining polynomial phase signal parameter estimates", IEEE Transactions on Aerospace and Electronic Systems, vol. 46, no. 3, pp. 978-987, 2010.

[36] D. Giffith, J. Gonzalez, and G. Arce, "Robust time frequency representiations for signals in alpha-stable noise using fractional lower-order statistics [C]", IEEE Signal Processing Worshop on High-Order Statistics, vol. 2, pp. 1233-1236, 1997.

[37] G. R. Arce, Nonlinear signal processing: a statistical approach, John Wiley \& Sons, 2005.
[38] S. Koike, "Analysis of normalized correlation algorithm for adaptive lters in impulsive noise environments", 2011 19th European Signal Processing Conference, pp. 141-145, 2011.

[39] M. Mahot, F. Pascal, P. Forster, and J. P. Ovarlez, "Robust covariance matrix estimates with attractive asymptotic properties", 2011 4th IEEE International Workshop on Computational Advances in Multi-Sensor Adaptive Processing (CAMSAP)", pp. 305-308, 2011.

[40] B. Boashash, "Estimating and interpreting the instantaneous frequency of a signal. II. Algorithms and applications", Proceedings of the IEEE, vol. 80, no. 4, pp. 540-568, 1992.

41] LJ. Stanković, I. Djurović, S. Stanković, M. Simeunović, S. Djukanović, and M. Daković, "Instantaneous frequency in timefrequency analysis: Enhanced concepts and performance of estimation algorithms", Digital Signal Processing, vol. 35, pp. 1-13, 2014.

[42] LJ. Stanković, S. Stanković, and M. Daković, "From the STFT to the Wigner distribution" [lecture notes], IEEE EEE Signal Processing Magazine, vol. 31, no. 3, pp. 163-174, 2014.

[43] D. Middleton, "Non-Gaussian noise models in signal processing for telecommunications: new methods an results for class A and class B noise models", IEEE Transactions on Information Theory, vol. 45, no. 4, pp. 1129-1149, 1999.

Received 19 April 2020 